\title{
Current standards for diagnosis and treatment of syphilis: selection of some practical issues, based on the European (IUSTI) and U.S. (CDC) guidelines
}

\author{
Maciej Pastuszczak, Anna Wojas-Pelc \\ Department of Dermatology, Jagiellonian University Medical College, Krakow, Poland \\ Head: Prof. Anna Wojas-Pelc MD, PhD
}

Postep Derm Alergol 2013; XXX, 4: 203-210

DOI: 10.5114/pdia.2013.37029

\begin{abstract}
Syphilis continues to be an important epidemiologic problem. For a few years a steady increase in the incidence of this sexually transmitted disease has been observed. Advances in medical science obligate the doctor to use only such diagnostic and therapeutic approaches that are scientifically proven. Based on the European (IUSTI) and U.S. (CDC) guidelines, in this manuscript, we present some selected practical issues concerning diagnosis and treatment of syphilis. We truly hope that the present review will help all doctors taking care of syphilitic patients to systematize the current knowledge.
\end{abstract}

Key words: syphilis, diagnosis, treatment, guidelines, CDC, IUSTI.

\section{Introduction}

Syphilis is a sexually transmitted disease found only in humans, which is caused by a spirochete (Treponema pallidum). Infection is characterized by a wide symptomatology, which makes the diagnosis difficult when based solely on the clinical picture [1]. The disease continues to be a significant epidemiological problem [2, 3]. The World Health Organization estimates that each year in the world there are about 11 million new cases [4]. Since 2000 in most European countries and the North America a steady increase in the incidence of syphilis has been observed [5].

Two documents that contain guidelines for diagnosis and management of patients with syphilis have been already published. The first one was developed by the International Union against Sexually Transmitted Infections (IUSTI, last updated in 2008) [6] and the second one was developed by the U.S. Centers for Disease Control and Prevention (CDC, last updated in 2010) [7]. The guidelines developed by the CDC and IUSTI are not in conflict.

Advances in medical science obligate doctors to take only these diagnostic and therapeutic approaches that have been proven to be effective. The CDC and IUSTI guidelines are based on scientific research. An appropriate level of evidence was assigned to each therapeutic approach. It should also be noted that following the guidelines is a line of defense in all disputes.

\section{Diagnosis of syphilis}

According to CDC and IUSTI guidelines, blood serological tests for syphilis (in addition to clinical features) remain the diagnostic standard. At least one treponemal antigen test and one non-treponemal antigen test are sufficient to confirm syphilis. The most widely used and recommended non-treponemal antigen tests (also referred to as cardiolipin antigen, or non-specific ones) are Venereal Disease Research Laboratory (VDRL) and Rapid Plasma Reagin (RPR) [8]. Non-treponemal antigen test results should be given quantitatively (i.e. titers, for example, $1: 16,1: 32$ ). It should be emphasized that the titers of non-treponemal antigen tests correlate with the disease activity. Non-treponemal test antibody titers should be also used to assess treatment response. The required decline in non-treponemal test titers after treatment, compared to baseline, is a marker of the correct response to the treatment. The VDRL and RPR are equally valid assays, but quantitative results from the two tests cannot be compared directly. In practice, this means that the non-treponemal antigen test used to assess treatment response

Address for correspondence: Maciej Pastuszczak MD, PhD, Department of Dermatology, Jagiellonian University Medical College, 8 Skawińska St, 31-066 Krakow, Poland, phone: +48 602228 796, e-mail: mpastuszczak@wp.p Received: 15.05.2013, accepted: 23.06.2013. 
should be the same as the one applied for diagnostic purposes.

Treponemal tests (otherwise referred to as specific ones) are the second type of diagnostic tests necessary for the diagnosis of syphilis. Besides 'classic' ones, which are widely used, such as Treponema pallidum haemagglutination test (TPHA), Fluorescent Treponemal Antibody Absorption test (FTA-ABS), Treponema pallidum Particle Agglutination test (TPPA), there are some new tests - i.e. immunoassays (Enzyme Immunoassays - EIAs). Most of EIAs detect total anti-treponemal antibodies (IgG and IgM). Currently, there are several commercially available kits such as the ICE Syphilis ${ }^{\circledR}$, Trepanostica ${ }^{\circledR}$, Pathozyme Syphilis ${ }^{\circledR}$, Enzygnost ${ }^{\circledR}$ Syphilis, Syphilis Bioelisa ${ }^{\circledR}$ and TrepChek ${ }^{\circledR}$. The sensitivity and specificity of these assays range between $64 \%$ and $95 \%$ [9]. All are equally valid. It is not necessary to verify results of the immunoassays with other tests. Noteworthy, treponemal test antibody titers should not be used to assess treatment response. Capita Syphilis $M^{\circledR}$ is a special immunoassay that detects IgM antibodies only. It may be used in the diagnosis of congenital syphilis and early syphilis formerly defined as 'serum-negative' (when serological tests are negative). In cases of such early syphilis, quantitative Capita Syphilis $M^{\circledR}$ may be used to assess treatment response.

Both CDC and IUSTI no longer recommend FTA for the diagnosis of syphilis as being very low specific for Treponema pallidum $[6,7]$.

\section{Screening tests for syphilis}

Treponemal antigen tests, such as TPHA or FTA-ABS, are currently recommended as screening tests for syphilis. Such a strategy will identify both persons with previous treatment for syphilis and persons with untreated or incompletely treated syphilis. On the other hand, it has been proven that in the general population, false positive treponemal tests occur less frequently than false positive non-treponemal tests. Therefore, in a smaller percentage of cases it is necessary to verify the diagnosis with nontreponemal antigen tests. It significantly reduces costs of the diagnostic procedure [10].

\section{Diagnostic scheme}

Figure 1 shows a diagnostic scheme recommended by the CDC and IUSTI (prepared by the authors based on the recommendations) $[6,7]$. Persons with a positive treponemal test (screening) should have a standard non-treponemal test with titer (e.g. VDRL). If both tests are positive, syphilis can be diagnosed. The patient's medical history and clinical symptoms should be, however, taken into account. After the treatment of syphilis, even several years afterwards, the patient may have positive treponemal and non-treponemal tests (at low titers). If the treponemal test (screening) is positive and the non-treponemal test is negative, then the laboratory should perform a different treponemal test (preferably one based on different antigens than the original test). If the second

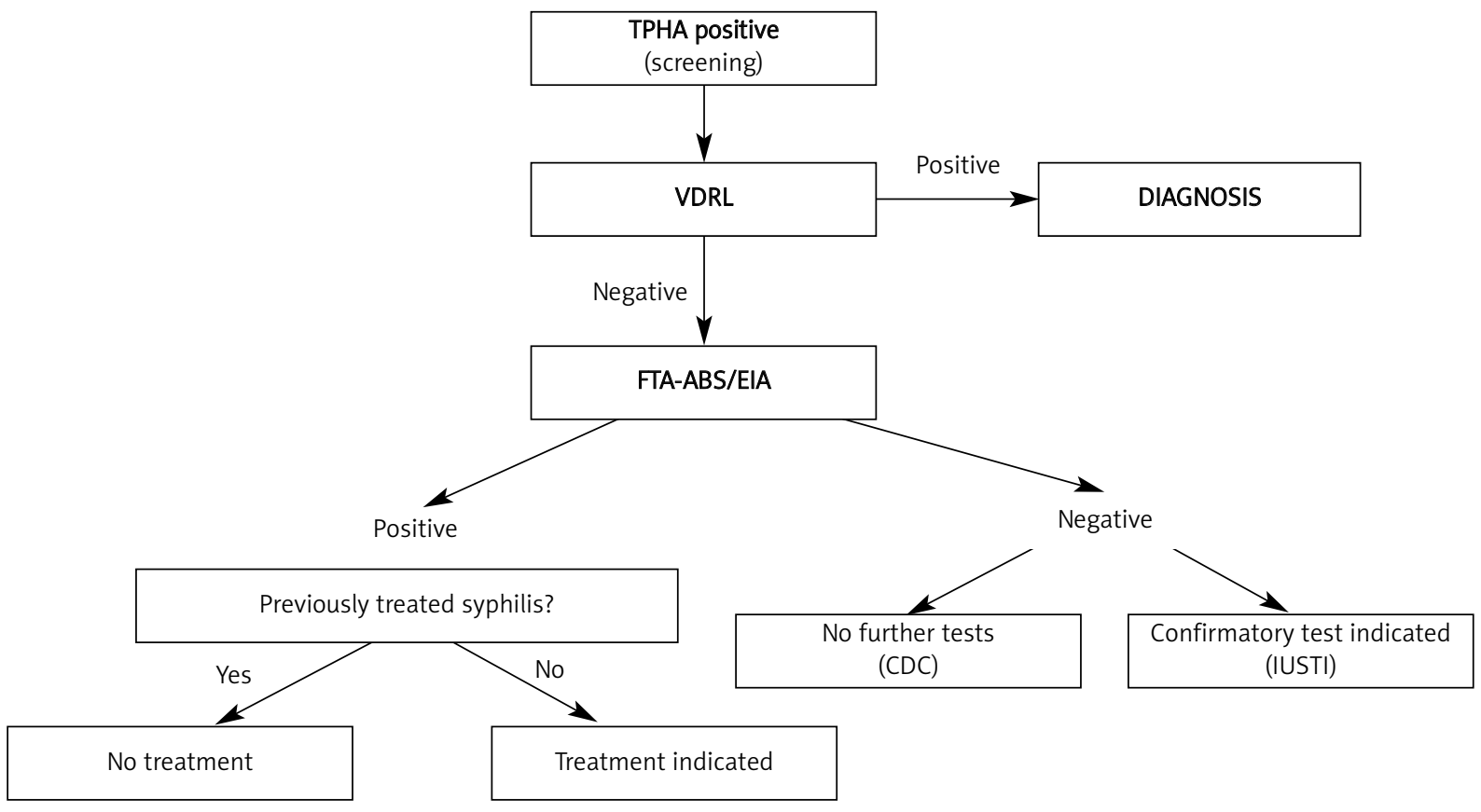

Fig. 1. Syphilis diagnostic scheme 
treponemal test is positive, persons with a history of previous treatment will require no further management unless sexual history suggests likelihood of re-exposure, and the patient has no signs and symptoms consistent with early syphilis (e.g. primary lesion). Patients without a previous history of treatment for syphilis should be offered treatment. Unless the history or results of a physical examination suggest a recent infection, such persons should be treated for late latent syphilis (see below).

If the second treponemal test is negative (first treponemal test positive, second non-treponemal negative), according to CDC further evaluation or treatment is not indicated. However, IUSTI recommends a supplementary confirmatory test in such situation. It should be the IgG immunoblot test using recombinant antigens (such as p44, p47, p17, p15). The FTA-ABS may be used as a supplementary test in certain circumstances, e.g. if it has not been used before as the second treponemal test and the laboratory is highly specialized with a large volume of confirmatory testing, where the quality of reagents and reproducibility of the test can be assured (Figure 1).

\section{Tests for monitoring the effect of treatment}

Non-treponemal antigen tests, such as VDRL and RPR, are almost exclusively recommended for monitoring the serological response to treatment. The titer determined on a blood specimen taken on the day of treatment gives the baseline for measuring a decrease in titer. In certain situations, monitoring after the treatment can be based on the quantitative test results of Capita Syphilis $M^{\circledR}$.

\section{Neurosyphilis: a diagnostic challenge}

The clinical picture of neurosyphilis has substantially changed in the past two decades [11]. Nowadays, 'paralysis progressiva' and 'tabes dorsalis' are seen rarely. Centers for Disease Control and Prevention (CDC) emphasizes that more and more cases of neurosyphilis can manifest as (1) meningitis, (2) ischemic stroke, especially in people under 40 years of age, (3) rapidly progressive dementia, especially in young patients, (4) impaired proprioception, and (5) hearing and sight disturbances, especially unexplained uveitis or sudden hearing loss which cannot be explained otherwise. The European and U.S. guidelines recommend searching for the above-mentioned neurological manifestations in patients with syphilis. It is also highly recommended to perform syphilis testing in patients being admitted to neurology departments due to the above-mentioned neurological symptoms.

No single diagnostic test can be used to diagnose neurosyphilis. The standard serologic test for cerebrospinal fluid (CSF) is VDRL [7]. Noteworthy, the other non-treponemal tests such as RPR and USR are not recommended for CSF. It is emphasized that the VDRL in CSF is highly specific. A positive result, in the absence of CSF contamination with blood, confirms the diagnosis. However, a negative result does not exclude neurosyphilis. CSF-VDRL may be negative in 30-70\% of neurosyphilis cases [12]. Both IUSTI and the CDC highlight that in the cases of the negative CSF VDRL, other tests should be taken into consideration, such as treponemal assays, CSF cell count, protein and glucose levels. Treponemal tests performed in CSF (TPHA, FTA-ABS, EIA) are highly sensitive but nonspecific for the neurosyphilis diagnosis. This means that the negative results exclude neurosyphilis, but the positive result does not confirm the diagnosis. The CSF white cell count cutoff values, which may suggest neurosyphilis, have been established on the $\geq 5$ cells/ $\mathrm{mm}^{3}$ in immunocompetent patients with syphilis and $\geq 20$ cells $/ \mathrm{mm}^{3}$ in HIV-positive patients. Neurosyphilis may be also associated with the CSF protein concentration higher than $45 \mathrm{mg} / \mathrm{dl}$ [13] and the CSF glucose levels of less than $2.72 \mathrm{mmol} / \mathrm{l}$ [14]. So far, there has been no consensus on how many of the above-mentioned additional criteria must be stated for neurosyphilis diagnosis, when the CSF VDRL is negative. However, most experts consider that pleocytosis (i.e. elevated CSF cell count) is a necessary condition in all instances.

\section{Screening for other sexually transmitted infections}

Both the CDC and IUSTI emphasize that all patients with a diagnosis of syphilis should be offered: (1) anti-HIV, (2) anti-HCV, and (3) HbsAg tests. Depending on the time from the exposure it may reasonable to advise the patient to repeat the tests after 3 months $[6,7]$.

\section{Treatment: general remarks}

In the experimental studies it has been shown that a penicillin level of above $0.018 \mathrm{mg} / \mathrm{l}$ in blood and CSF should be considered treponemicidal [15]. Duration of the treponemicidal level should be at least 7-10 days to cover a number of division times of treponemes. Benzathine penicillin at a single dose of 2.4 million units provides a treponemicidal concentration for up to 3-4 weeks (2123 days) [16]. Duration of treponemicidal concentration of procaine penicillin after a single dose is not precisely defined. There is, however, no doubt that it is significantly shorter. It has been suggested that it may be even less than $24 \mathrm{~h} \mathrm{[6].}$

\section{Assessing the duration of asymptomatic disease}

Diagnosis of primary and secondary syphilis is based on clinical features. Latent syphilis is defined as a stage not accompanied by clinical symptoms but the patient has a positive serological test. Because of different therapeutic approaches to early latent syphilis and late latent syphilis it is important to distinguish these two stages of infection. 
Table 1. Criteria required for the diagnosis of early latent syphilis

within the last year $\left\{\begin{array}{c}\text { Positive syphilis blood testing and lack of clinical symptoms } \\ \text { and }\end{array}\right.$

It was arbitrarily assumed that early syphilis is an infection that lasts not longer than a year according to the CDC and not more than 2 years by IUSTI. Late syphilis is defined as a disease lasting over 1 or 2 years, respectively. Table 1 shows the criteria required for the diagnosis of early latent syphilis (prepared by the authors based on the CDC guidelines). All patients who (1) do not have at least one criterion specified in Table 1, (2) have positive serological tests in blood, (3) and do not present clinical symptoms, should be treated for late latent syphilis.

\section{Syphilis treatment recommendations are based on the studies of a certain level of evidence}

Each therapeutic recommendation for different stages of syphilis (i.e. drug regimen, duration of treatment) was assigned an appropriate level of evidence. Table 2 gives a brief description of research methodology along with the associated level of evidence. Randomized controlled trials have been classified as la and Ib. They are considered as the most reliable ones.

Table 2. Research methodology description and associated level of evidence

\begin{tabular}{cl}
\hline $\begin{array}{c}\text { Level of } \\
\text { evidence }\end{array}$ & \multicolumn{1}{c}{ Description } \\
\hline Ia & Meta-analysis of randomized control trials \\
\hline Ib & At least one randomized control trial \\
\hline Ila & $\begin{array}{l}\text { At least one well-designed study without } \\
\text { randomization }\end{array}$ \\
\hline Ilb & $\begin{array}{l}\text { At least one well-designed quasi-experimental } \\
\text { study }\end{array}$ \\
\hline III & $\begin{array}{l}\text { At least one well-designed non-experimental } \\
\text { descriptive study }\end{array}$ \\
\hline IV & $\begin{array}{l}\text { Expert committee reports, opinions and/or } \\
\text { experience }\end{array}$ \\
\hline
\end{tabular}

\section{Treatment of primary, secondary and early latent syphilis}

Table 3 shows the recommended management of primary, secondary and early latent syphilis. Benzathine penicillin at a dose of 2.4 million units administered as a single intramuscular injection remains the treatment of choice. Noteworthy, alternative medications are recommended only in cases of penicillin allergy or parenteral treatment refusal. However, it should be carefully noted in the patient's medical record [6]. Data on the efficacy of ceftriaxone are derived from a single randomized trial [17]. It should be however stressed that until now, the optimal dose and duration of ceftriaxone therapy have not been defined. Moreover, there is a significant cross reaction between cephalosporins and penicillin. Thus, using ceftriaxone as an alternative in patients allergic to penicillin is limited. Efficacy of azithromycin has also been shown in randomized trials. It was recommended at a dose of 2 g, given only once, orally [18]. However, intrinsic resistance to azithromycin has been described since 2004. Thus, azithromycin is no longer recommended [19].

\section{Treatment of late latent syphilis and syphilis of unknown duration}

Recommended medication for late latent syphilis and syphilis of unknown duration is shown in Table 4. Benzathine penicillin and procaine penicillin are the first-line therapies. However, the duration of therapy is longer when compared to early syphilis. The IUSTI and CDC suggest that in cases of allergy to penicillin, desensitization should be considered as the evidence base for the use of non-penicillin regimens, which is relatively weak in late latent syphilis and syphilis of unknown duration.

\section{Treatment of tertiary syphilis}

Treatment of choice in tertiary syphilis is benzathine penicillin at a dose of 2.4 million units, given once a week for 3 consecutive weeks. 
Table 3. Treatment of primary, secondary and early latent syphilis

\begin{tabular}{lccc}
\hline Drug & Dose, administration & Treatment duration & Level of evidence \\
\hline Benzathine penicillin & 2.4 million units, IM & Single dose & $\mathrm{lb}$ \\
\hline Procaine penicillin & 600000 units once daily, IM & $10-14$ days & $\mathrm{Ilb}$ \\
\hline \multicolumn{4}{c}{ Alternatives } \\
\hline Doxycycline & $200 \mathrm{mg}$ once daily, PO & 14 days & $\mathrm{III}$ \\
\hline Tetracycline & $500 \mathrm{mg} 4 \times$ daily, PO & 14 days & $\mathrm{III}$ \\
\hline Erythromycin & $500 \mathrm{mg} 4 \times$ daily, PO & 14 days & $\mathrm{IV}$ \\
\hline Azithromycin* & $2 \mathrm{~g}, \mathrm{PO}$ & Single dose & $\mathrm{Ib}$ \\
\hline Ceftriaxone* & $500 \mathrm{mg}$ once daily, IM & 10 days & $\mathrm{lb}$ \\
\hline *See the text & & &
\end{tabular}

Table 4. Treatment of late latent syphilis and syphilis of unknown duration

\begin{tabular}{lccc}
\hline Drug & Dose, administration & Treatment duration & Level of evidence \\
\hline Benzathine penicillin & 2.4 million units $1 \times$ week, IM & 3 consecutive weeks & III \\
\hline Procaine penicillin & 600000 units once daily, IM & $17-21$ days & III \\
\hline \multicolumn{4}{c}{ Alternatives } \\
\hline Doxycycline & $200 \mathrm{mg}$ once daily, PO & $21-28$ days & $\mathrm{IV}$ \\
\hline Tetracycline & $500 \mathrm{mg}$ 4× daily, PO & 28 days & $\mathrm{IV}$ \\
\hline Erythromycin & $500 \mathrm{mg}$ 4× daily, PO & 28 days & $\mathrm{IV}$
\end{tabular}

Table 5. Treatment of neurosyphilis

\begin{tabular}{lccc}
\hline Drug & Dose, administration & Treatment duration & Level of evidence \\
\hline Crystalline penicillin & 12-24 million units daily, & $18-21$ days (IUSTI) & III \\
& 3-4 million units every 4 h, I.V. & $10-14$ days (CDC) & IIb \\
\hline Procaine penicillin & $\begin{array}{l}1.2-2.4 \text { million units once daily, } \\
\text { IM, plus probenecid 500 mg 4× daily, PO }\end{array}$ & $\begin{array}{c}10-17 \text { days (IUSTI) } \\
10-14 \text { days (CDC) }\end{array}$ & \\
\hline \multicolumn{2}{c}{ Alternatives } \\
Doxycycline & $200 \mathrm{mg} 2 \times$ daily, PO & 28 days & IV \\
\hline
\end{tabular}

\section{Treatment of neurosyphilis}

Neurosyphilis may occur both in early and late syphilis. Table 5 shows the recommended management of neurosyphilis. The use of non-penicillin regimens is weak. Thus, in cases of penicillin allergy, desensitization should be considered.

\section{Treatment of syphilis in HIV-positive patients}

Both the CDC and IUSTI emphasize that until now, there has been no sufficient scientific evidence to treat HIV coinfected syphilitic patients otherwise than immunocompetent patients $[20,21]$. An increased risk of the central nervous system (CNS) involvement and treatment failure may occur in patients with HIV who are in significant im-

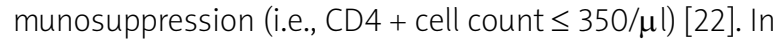

these patients, it seems reasonable to perform an immediate lumbar puncture. Further management should be based on the results of CSF examination.

\section{Follow-up after treatment}

Correct response to treatment is defined as: (1) resolution of clinical symptoms, and (2) at least a 4-fold decline in the titer of non-treponemal tests (e.g. VDRL), when compared to baseline: (a) at 6 months after the treatment (for primary, secondary and early latent syphilis), (b) within 6-12 months after treatment (for syphilitic patients with HIV co-infection), or (c) within 1224 months after treatment (for late latent syphilis and syphilis of unknown duration) [6, 7]. Table 6 shows the recommended (both according to the CDC and IUSTI) fre- 
Table 6. Frequency of follow-up appointments after completing syphilis treatment

\begin{tabular}{|c|c|c|c|c|c|c|c|}
\hline Months from the treatment end & 1 & 2 & 3 & 6 & 9 & 12 & 24 \\
\hline \multicolumn{8}{|c|}{ Primary, secondary and early latent syphilis } \\
\hline CDC 2010 & & & & $x$ & & $x$ & \\
\hline IUSTI 2008 & $x$ & $x$ & $x$ & $x$ & & $x$ & \\
\hline \multicolumn{8}{|c|}{ Late latent syphilis and syphilis of unknown duration } \\
\hline CDC 2010 & & & & $x$ & & $x$ & $x$ \\
\hline IUSTI 2008 & $x$ & $x$ & $x$ & $x$ & $x$ & $x$ & $x$ \\
\hline
\end{tabular}

"X" means that at this time follow-up appointment is recommended, CDC - Centers for Disease Control and Prevention, IUSTI - International Union against Sexually Transmitted Infections

quency follow-up appointments after treatment for different stages of syphilis. There is no consensus on followup after treatment of tertiary syphilis. It appears, however, that the follow-up appointments should be more frequent and take place for a longer period of time.

Inadequate response to treatment is defined as: (1) persistence of clinical symptoms, (2) 4-fold increase in titer of the non-treponemal test (e.g. VDRL) and (3) absence of at least a 4-fold decline in titer of the non-treponemal test ascertained in certain time periods (defined previously for different groups of syphilitic patients; see above). Persistence of symptoms (group 1) and a 4-fold increase in titer of the non-treponemal test (group 2) are almost always associated with re-infection or treatment failure. However, lack of at least a 4-fold decline in titer of the non-treponemal test may affect as many as $15 \%$ of immunocompetent patients and may be not linked to re-infection or treatment failure. Figures $2 \mathrm{~A}$ and $\mathrm{B}$ (prepared by the authors based on the guidelines) present the patient care scheme in cases of inadequate treatment response. To sum up, almost always re-treatment is indicated. The therapeutic scheme (i.e., treatment with penicillin intramuscularly or intravenously) should be chosen according to results of CSF examination. Noteworthy, there is no consensus on the management of patients who would not achieve at least a 4-fold decrease in titer of non-treponemal tests.

(1) Persistence of clinical symptoms - group 1

When after treatment:

(2) 4-fold increase in titer of non-treponemal test (VDRL, RPR) - group 2

(3) Lack of at least 4-fold decline in titer of non-treponemal at: (a) 6 month after completing the treatment (primary, secondary and early latent syphilis), (b) 12-24 month after completing the treatment (late latent syphilis or syphilis of unknown duration) - group 3

a. Primary, secondary and early latent syphilis CSF examination, and again HIV testing

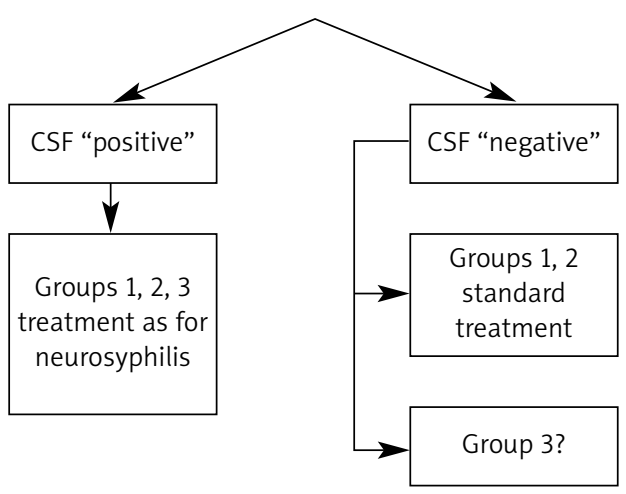

b. Late latent syphilis and syphilis of unknown duration CSF examination, again HIV testing and re-treatment (always)*

*Treatment scheme according to CSF examination results

CSF - cerebrospinal fluid, CSF "positive" - abnormalities in CSF examination consistent with neurosyphilis diagnosis (see the text), CSF "negative" - no CSF abnormalities or CSF abnormalities not consistent with neurosyphilis diagnosis (see the text)

Fig. 2. Patient care scheme in cases of inadequate treatment response 


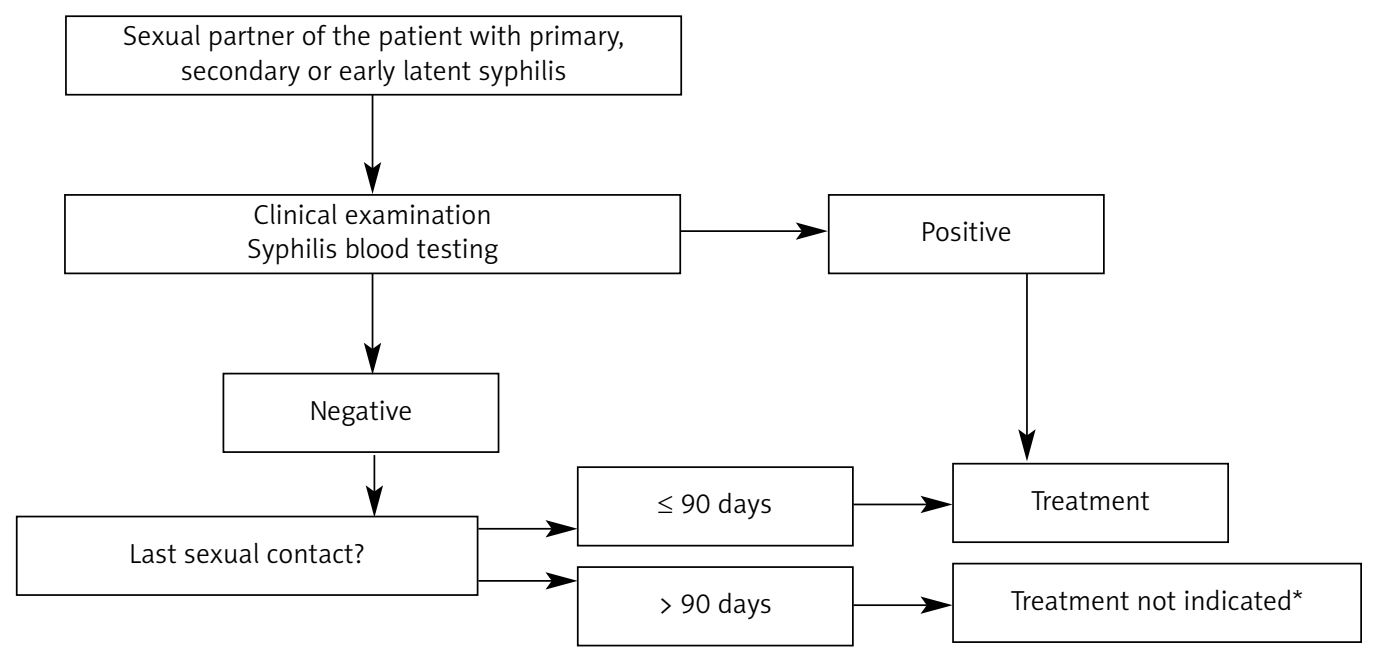

${ }^{*}$ Clinical examination and syphilis blood testing again after 6 weeks and 3 months

Fig. 3. Sex partner care scheme

Some experts recommend that despite normal results of CSF examination, re-treatment should be initiated.

Both IUSTI and CDC note that follow-up after neurosyphilis treatment is based on repeated examination of CSF. The first follow-up lumbar puncture should be performed 6 months after the end of treatment. The correct treatment response is considered as a decrease in the number of white blood cells (WBC) in CSF (i.e. $<5 / \mathrm{mm}^{3}$ in immunocompetent patients and $<20 / \mathrm{mm}^{3}$ in HIV-positive ones). When the WBC count has not decreased to the desired value, the treatment must be repeated. Next CSF examinations should be done after 6 months.

\section{Diagnosis and treatment of sex partners}

Both the CDC and IUSTI particularly stress the importance of proper diagnosis and management of sexual partners of the patient with syphilis. It has been estimated that up to $60 \%$ of sexual contacts of patients with syphilis may be infected. A detailed sex history should be taken from the syphilitic patient with particular reference to persons with whom the patient had oral, vaginal and anal intercourses (both with and without protection). Sexual partners of infected patients should be considered at risk if they have had sexual contact with the patient within 3 months for patients diagnosed with primary syphilis. If the infected patient has been diagnosed as 'early syphilis', medical advice should be given to all sex partners within one year (CDC) or 2 years (IUSTI). Figure 3 (prepared by the authors based on the guidelines) presents a sex partner care scheme. It should be noted that persons exposed within 90 days preceding the diagnosis of primary, secondary and early latent syphilis in a sex partner should be treated even if the results of the clinical and serological examination are negative. Management for early syphilis should be initiated.

\section{Conclusions}

Syphilis remains to be a serious epidemiological problem. Advances in medical science obligate doctors to apply proven effective diagnostic and therapeutic procedures. Following the guidelines remains the only line of defense for a doctor in cases of a dispute. Despite a great number of new treponemal tests, non-treponemal tests are still very important. Non-treponemal tests are necessary for the final diagnosis, they are only recommended for the assessment of treatment response and are of the highest specificity for the diagnosis of neurosyphilis. Penicillin remains the drug of choice in the treatment of all forms of syphilis. Diagnosis, treatment, and follow-up of neurosyphilis are difficult. It is suggested that such patients should be referred to the tertiary reference centers. The treatment scheme for syphilitic patients with HIV co-infection should be the same as for immunocompetent patients. Special attention should be paid to the notification, diagnosis and treatment of sexual partners of the infected patients.

\section{References}

1. Pastuszczak M, Wozniak W, Jaworek A, et al. Pityriasis lichenoides-like secondary syphilis and neurosyphilis in a HIV infected patient. Postep Derm Alergol 2013; 30: 127-30.

2. Karlinska-Jachowska M, Chmielnicki P, Dziankowska-Bartkowiak B, et al. Syphilis - issue of the 21st century. Postep Derm Alergol 2007; 24: 233-7.

3. Jakubowicz O, Żaba R, Czarnecka-Operacz M. Serological tests for syphilis performed in the Sexually Transmitted Diseases Diagnostic Laboratory in Poznań during 2000-2004. Postep Derm Alergol 2011; 28: 30-5

4. Gerbase AC, Rowley JT, Heymann DH, et al. Global prevalence and incidence estimates of selected curable STDs. Sex Transm Infect 1998; 74 (suppl 1): S12-6. 
5. Fenton KA, Breban R, Vardavas R, et al. Infectious syphilis in high-income settings in the 21st century. Lancet Infect Dis 2008; 8: 244-53.

6. French P, Gomberg M, Janier M, et al. IUSTI: 2008 European Guidelines on the Management of syphilis. Int J STD AIDS 2009; 20: 300-9.

7. Workowski KA, Berman S. Sexually transmitted diseases guidelines, 2010. MMWR 2010; 59: 1-116.

8. Young $\mathrm{H}$. Guidelines for serological testing for syphilis. Sex Transm Infect 2000; 76: 403-5.

9 Schmidt BL, EdjlalipourM, Luger A. Comparative evaluation of nine different enzyme-linked immunosorbent assays for determination of antibodies against Treponema pallidum in patinets with primary syphilis. J Clin Microbiol 2000; 38: 1279-82.

10. Pope V. Use for treponemal tests to screen for syphilis. Infect Med 2004; 21: 399-402.

11. Chahine LM, Khoriaty RN, Tomford WJ, et al. The changing face of neurosyphilis. Int J Stroke 2011; 6: 136-43.

12. Golden MR, Marra CM, Holmes KK. Update on syphilis. Resurgence of an old problem. JAMA 2003; 11: 1510-4.

13. Ghanem KG. Neurosyphilis: a historical perspective and review. CNS Neurosc Ther 2010; 16: e157-68.

14. Pastuszczak M, Wojas-Pelc A, Jaworek A. Association of CSF glucose concentration with neurosyphilis diagnosis. Cent Eur J Med 2013; 8: 48-51.

15. Rolfs RT. Treatment of syphilis. Clin Infect Dis 1995; 20 (Suppl 1): S23-38.

16. Idsoe O, Guthe T, Willcox RR. Penicillin in the treatment of syphilis. The experience of three decades. Bull WHO 197; 47: 1-68.

17. Hook EW, Roddy RR, Handsfield HH. Ceftriaxone therapy for incubating and early syphilis. J Infect Dis 1988; 158: 881-4.

18. Riedner G, Rusizoka M, Todd J, et al. Single-dose azithromycin versus penicillin $\mathrm{G}$ benzathine for the treatment of early syphilis. N Engl J Med 2005; 353: 1236-44.

19. Mitchell SJ, Engelman J, Kent CK, et al. Azithromycin resistant syphilis infection: San Francisco, California, 2000-2004. Clin Infect Dis 2006; 42: 337-45.

20. Rompalo AM, Joesoef MR, O'Donnell JA, et al. Clinical manifestation of early syphilis by HIV status and gender. Results of the Syphilis and HIV Study. Sex Transm Dis 1997; 28: 158-65.

21. Rolfs RT, Joesoef MR, Hendershot EF, et al. A randomized trial of enhanced therapy for early syphilis in patients with and without HIV infection. N Engl J Med 1997; 337: 307-14.

22. Marra C, Maxwell CL, Smith SL, et al. Cerebrospinal fluid abnormalities in patients with syphilis: association with clinical and laboratory features. J Infect Dis 2004; 189: 369-76. 